\title{
HOLOMORPHIC SYMPLECTIC GEOMETRY AND ORBIFOLD SINGULARITIES*
}

\author{
MISHA VERBITSKY ${ }^{\dagger}$
}

\begin{abstract}
Let $G$ be a finite group acting on a symplectic complex vector space $V$. Assume that the quotient $V / G$ has a holomorphic symplectic resolution. We prove that $G$ is generated by "symplectic reflections", i.e. symplectomorphisms with fixed space of codimension 2 in $V$. Symplectic resolutions are always semismall. A crepant resolution of $V / G$ is always symplectic. We give a symplectic version of Nakamura conjectures.
\end{abstract}

\section{Introduction.}

1.1. Symplectic desingularizations in algebraic geometry and representation theory. Let $V$ be a complex vector space, and $G$ a finite group acting on $V$ by linear transformations. The variety $X=V / G$ is usually singular, and this paper deals with its desingularizations (also called resolutions). A resolution of $X$ is a proper birational map $\pi: \widetilde{X} \longrightarrow X$ such that $\tilde{X}$ is smooth, and $\pi$ is an isomorphism outside of singularities of $X$.

A singularity of the type $V / G$ is called a quotient or orbifold singularity.

The crepant resolutions of $X$ are resolutions $\pi: \tilde{X} \longrightarrow X$ such that the canonical class of $\widetilde{X}$ is obtained as a pullback of a canonical class of $X$ (see 2.1).

EXAMPLE 1.1. The Hilbert scheme of $n$ points on $\mathbb{C}^{2}$ provides a crepant resolution of the quotient $\left(\mathbb{C}^{2}\right)^{n} / S_{n}$ of $\left(\mathbb{C}^{2}\right)^{n}$ by the natural action of the symmetric group $S_{n}$ (this is well known; see e. g. [N]).

The crepant resolutions of quotient singularities in dimension 3 and more became a focus of intense study after the paper [IR] of Y. Ito and M. Reid, because of their relations with physics and with the theory of Hilbert schemes ([IN]). For a history of these questions and their relevance to the mirror symmetry, see [R] and [BD].

Another reason to study the crepant resolutions comes from the holomorphic symplectic geometry and representation theory. Suppose that a complex vector space space $V$ is equipped with a $\mathbb{C}$-valued symplectic form, and $G$ acts on $V$ by symplectic transformations. The desingularization $\widetilde{X} \longrightarrow X$ is called a symplectic resolution if $\widetilde{X}$ is holomorphic symplectic, and the holomorphic symplectic form on $\widetilde{X}$ is lifted from $X$ (see 2.3). Clearly, the symplectic resolutions are always crepant. It turns out that, conversely, any crepant resolution of $(V / G)$ is symplectic (2.4). Symplectic resolutions were studied by R. Bezrukavnikov and V. Ginzburg (1998, unpublished), who worked in the following situation. Consider a semi-simple Lie algebra $\mathfrak{g}$ over $\mathbb{C}$, and its Cartan subalgebra $\mathfrak{h} \subset \mathfrak{g}$. Bezrukavnikov and Ginzburg considered the space $\mathfrak{h} \oplus \mathfrak{h}^{*}$ (a direct sum of a Cartan algebra with its dual). Clearly, $\mathfrak{h} \oplus \mathfrak{h}^{*}$ is a symplectic vector space, equipped with a natural action of the Weyl group $W$ of $\mathfrak{g}$. They suggested that the variety $\mathfrak{h} \oplus \mathfrak{h}^{*} / W$ admits a natural symplectic desingularization, and this desingularization is hyperkähler. This is true for the case $\mathfrak{g}=\mathfrak{s l}(n)$, because in this case

$$
\left(\mathfrak{h} \oplus \mathfrak{h}^{*}\right) / W \cong\left(\mathbb{C}^{2}\right)^{n} / S_{n}
$$

\footnotetext{
*Received April 20, 1999; accepted for publication November 9, 1999.

†11-1-83 Orekhovy blvrd, Moscow, 115551, Russia (verbit@thelema.dnttm.rssi.ru). Research partially supported by CRDF grant RM1-2087.
} 
and the desingularization is provided by the Hilbert scheme (Example 1.1). The conjectural desingularizations of Bezrukavnikov and Ginzburg are quite important, because they generalize the usual Hilbert schemes.

A second example when Bezrukavnikov-Ginzburg conjecture is valid was invented by $A$. Kuznetsov, who considered the Lie algebras associated with the Dynkin diagrams $B_{n}, C_{n}$. The corresponding symplectic desingularization of $\mathfrak{h} \oplus \mathfrak{h}^{*} / W$ is birational to the Hilbert scheme of the total cotangent space $T^{*} \mathbb{C} P^{1}$ of $\mathbb{C} P^{1}$. Kuznetsov's construction is explained in more details in [KV2]. There is some indication that Bezrukavnikov-Ginzburg conjecture is not valid for the Dynkin diagrams $G_{2}, D_{n}$ $(n \geqslant 4)$ and $E_{n}(n=6,7,8)$.

Another reason to study the symplectic desingularization comes from the hyperkähler geometry. Consider a compact complex torus $T, \operatorname{dim}_{\mathbb{C}} T=2$, and its $n$-th Hilbert scheme of points $T[n]$. Let $A l b: T^{[n]} \longrightarrow T$ be the Albanese map. A generalized Kummer variety $K^{[n-1]}$ is defined as

$$
K^{[n-1]} \subset T^{[n]}, \quad K^{[n-1]}:=A l b^{-1}(0) .
$$

The variety $K^{[n-1]}$ is smooth and holomorphically symplectic ([Bea]). By Calabi-Yau theorem ([Y], [Bea]), the variety $K^{[n]}$ is equipped with a set of hyperkähler structures, parametrized by the Kähler cone. In [KV1], it was falsely claimed that, for a generic hyperkähler structure, $K^{[n]}$ has no subvarieties compatible with the hyperkähler structure (such subvarieties are called trianalytic, see [V1]). We mentioned above the simple agrument used by Kuznetsov to prove the existence of symplectic desingularizations of $\mathfrak{h} \oplus \mathfrak{h}^{*} / W$ for the Dynkin diagrams $B_{n}, C_{n}$ The same argument proves existence of trianalytic subvarieties of generalized Kummer varieties ([KV2], Theorem 6.10).

In [KV2] (Section 4), this topic was pursued further. It turns out that all trianalytic subvarieties of generalized Kummer varieties (at least, for generic hyperkähler structures) are isomorphic to symplectic desingularizations of a quotient of a compact torus by an action of a Weyl group. This establishes a very interesting relation between the Dynkin diagrams and hyperkähler geometry, and motivates the study of symplectic desingularization of quotient singularities.

1.2. Symplectic desingularizations and symplectic reflections. In this paper, we carry the argument used in [KV2] a step further, to obtain information about the structure of finite groups $G \subset S p(V)$ such that $V / G$ admits a symplectic desingularization. This is done as follows. Let $g \in \operatorname{End}(V)$ be a symplectomorphism of finite order. We say that $g$ is a symplectic reflection if

$$
\operatorname{codim}_{V}(\{x \in V \mid g(x)=x\})=2
$$

that is, the dimension of the fixed set of $g$ is maximal possible for non-trivial $g$ (see 3.1). This definition parallels that of complex reflections - a complex reflection is an endomorphism of finite order with fixed point set of codimension 1 . The main result of this paper is the following theorem.

THEOREM 1.1. Let $V$ be a symplectic vector space over $\mathbb{C}$, and $G \subset S p(V)$ a finite group of symplecic transformations. Assume that $V / G$ admits a symplectic resolution. Then $G$ is generated by symplectic reflections. 
Proof. This is 3.2, which is proven in Section 4.

This result is analogous to a well known theorem (3.5, Remark 3.3), stating that for any finite group of endomorphisms $G \subset$ End $V, V / G$ is smooth if and only if $G$ is generated by complex reflections. However, the "if" part in symplectic case is not proven and is likely false (see Conjecture 1.6).

In connection with 1.1, the following questions appear.

QUESTION 1.2. Is it possible to classify the groups generated by symplectic reflections?

A complete classification of groups generated by complex reflections was obtained in [ST] (Shephard and Todd, 1954).

The basic example of groups generated by symplectic reflections is the following.

EXAMPLE 1.3. Given an action of a group $G \subset$ End $W$ we consider the natural action of $G$ on $\operatorname{End}\left(W \oplus W^{*}\right)$. Clearly, $G$ acts on $W \oplus W^{*}$ preserving the standard symplectic structure. The action of $G$ on $W \oplus W^{*}$ is generated by symplectic reflections if and only if the action of $G$ on $W$ is generated by complex reflections (see the proof of 3.3). 1.3 .

The Weyl group acting on $\mathfrak{h} \oplus \mathfrak{h}^{*}$ (Subsection 1.1) is a special case of Example

REMARK 1.4. Not all the groups generated by symplectic reflections are provided by Example 1.3. Take, for instance, any finite subgroup $G \subset S L(V), \operatorname{dim} V=2$. Clearly, all non-trivial elements of $G$ are symplectic reflections. The group $G$ is obtained from Example 1.3 if and only if $G$ preserves a direct decomposition $V=$ $W_{1} \oplus W_{2}, \operatorname{dim} W_{1}=\operatorname{dim} W_{2}=1$. The finite subgroups of $S L(2)$ are well known, and for most of them such a decomposition does not exist.

Another question appearing in connection with 1.1 is the following

QUESTION 1.5. Let $G \subset$ End $V$ be a subgroup generated by symplectic reflections. Determine whether $V / G$ admits a symplectic resolution.

In the case $\operatorname{dim} V=2$, the answer is "always" by the classical results of Du Val; in the next non-trivial case $(\operatorname{dim} V=4)$ the answer is unknown already.

An example of a Weyl group of $G_{2}$ acting on $\mathfrak{h} \odot \mathfrak{h}^{*} \cong \mathbb{C}^{4}$ motivates the following version of Nakamura's conjecture $([\mathrm{R}])$.

ConjeCture 1.6. Let $G \subset S p(V)$ be a finite group acting on a symplectic $\mathbb{C}$ vector space, and $\tilde{X}:=$ Hilb $^{G} V$ the $G$-Hilbert scheme ([R]).

(i) Then $\tilde{X}$ is a smooth holomorphic symplectic orbifold with singularities in codimension $\geqslant 4$, and the natural map $\pi: \tilde{X} \longrightarrow V / G$ is an orbifold desingularization of $V / G$.

(ii) Moreover, for any crepant orbifold desingularization $\pi_{1}: \tilde{X}_{1} \longrightarrow V / G$ with $\operatorname{dim} \operatorname{Sing} \widetilde{X}_{1} \geqslant 4$, the orbifold $\widetilde{X}_{1}$ is diffeomorphic to $\widetilde{X}$.

The second part of Conjecture 1.6 is motivated by a result of Huybrechts $[\mathrm{H}]$ : birational holomorphic symplectic compact manifolds are diffeomorphic. 


\subsection{Contents.}

- The present Introduction is independent from the rest of this paper.

- In Section 2, we define symplectic desingularizations and state their main properties. Any crepant desingularization of a quotient singularity $V / G, G \subset$ $S p(V)$ is symplectic (2.4). Simplectic resolutions are semismall (2.7).

- In Section 3, we state our main result: if a symplectic desingularization of $V / G$ exists, then $G$ is generated by symplectic reflections (3.2). We illustrate this result by a proof of a classical theorem about groups generated by complex reflections.

- In Section 4, we give the proof of our main theorem. The proof is based on the following preliminary proposition (4.1). Let $\widetilde{X} \longrightarrow X$ be a resolution of a quotient-type singularity $X=V / G$. Then the manifold $\tilde{X}$ is simply connected.

2. Symplectic resolutions. Let $X$ be an irreducible complex analytic variety. A proper morphism $\pi: \quad \widetilde{X} \longrightarrow X$ is called a resolution of singularities, or a desingularization of $X$ if $\widetilde{X}$ is smooth and connected, and $\pi$ is an isomorphism outside of the set of singular points of $X$.

DEFINITION 2.1. In the above assumptions, let $X$ be a normal variety, and $U$ a non-singular part of $X$. Since $\pi$ is an isomorphism over $U$, we may consider $U$ as a subset in $\widetilde{X}$. Let $C \subset U$ be a divisor associated with the canonical class of $U$, and $\widetilde{C}$ a closure of $C$ in $\widetilde{X}$. We say that $\pi$ is crepant if the divisor $\widetilde{C}$ lies in the canonical class of $\widetilde{X}$.

In other words, crepant resolutions are those which preserve the canonical class.

DEFINITION 2.2. Let $V$ be a vector space, $G$ a finite group acting on $V$ by linear transformations, and $B \subset V$ a $G$-invariant open ball. In this case, we say that $B / G$ has orbifold singularities or singularities of quotient type.

DeFINITION 2.3. Let $\pi: \tilde{X} \longrightarrow X$ be a resolution of a quotient-type singularity $X=V / G$, where $V$ is a symplectic $\mathbb{C}$-vector space. Assume that $G$ acts on $V$ by linear transformations preserving the symplectic form: $G \subset S p(V)$. Consider the natural symplectic form $\Omega_{X}$ on $X$, defined outside of the singularities of $X$. Assume that the pullback $\pi^{*} \Omega$ can be extended to a holomorphic symplectic form on $\tilde{X}$. Then $\pi: \widetilde{X} \longrightarrow X$ is called a symplectic resolution of $X$.

REMARK 2.1. Clearly, symplectic resolutions are crepant.

The following preliminary theorem establishes the relationship between the symplectic and crepant resolutions. We shall not use it in this paper, but prove it here to validate the notion of a symplectic desingularization.

TheOREM 2.4. Let $X=V / G$ be a quotient of a symplectic vector space by a finite group $G \subset S p(V)$. Assume that $\pi: \widetilde{X} \longrightarrow X$ is a crepant resolution of singularities. Then $\pi$ is a symplectic resolution, in the sense of 2.3 .

Proof. Another proof of this theorem is given in [K], (Proposition 3.2), and in [Bea2], (Proposition 2.4).

Let $U \subset X$ be the set of all points where $\pi: \widetilde{X} \longrightarrow X$ is smooth, and $\widetilde{U}:=\pi^{-1}(U)$ the corresponding subset of $\tilde{X}$. Consider the symplectic form $\Omega_{X}$ as a section of the sheaf of holomorphic 2 -forms $\Omega_{U}^{2}$. Let $\Omega_{\widetilde{U}}:=\pi^{*} \Omega_{X}$ be its pullback. We need to show that the form $\Omega_{\widetilde{U}}$ can be extended to the whole $\widetilde{X}$. Consider a smooth Hermitian 
metric on $\widetilde{X}$, and let $h$ be the corresponding metric on $\Omega^{2} \widetilde{X}$. Then $\Omega_{\widetilde{U}}$ is a holomorphic section of a Hermitian vector bundle $\Omega_{\widetilde{U}}^{2}$ over $\widetilde{U}$. Such a section can be extended to a section of a bundle over $\tilde{X}$ unless it has singularities on the complement $\tilde{X} \backslash \tilde{U}$. To prove that $\Omega_{\widetilde{U}}$ can be extended to $\tilde{X}$, it remains to show that for any compact set $K \subset \widetilde{X}$ for every $x \in \widetilde{U} \cap K$, the Hermitian norm of $\Omega_{\widetilde{U}}$ is bounded

$$
h_{x}\left(\left.\Omega_{\tilde{U}}\right|_{x}\right)<C_{K}
$$

by some constant $C_{K}$ depending on $K$.

A symplectic form $\Theta$ in a Hermitian vector space $L$ can be naturally represented in the form

$$
\Theta=\sum_{i=0}^{n-1} \lambda_{i} z_{i+1} \wedge z_{i+2}
$$

for some orthonormal basis $z_{1}, \ldots, z_{2 n}$ in $L^{*}$, where $\lambda_{i}$ are non-negative real numbers. The numbers $\lambda_{i}$ are called the eigenvalues of $\Theta$; the set of eigenvalues is defined canonically by the symplectic form and the Hermitian form. Denote the eigenvalues by $\lambda_{i}(\Theta)$. Clearly, for any $x \in \widetilde{U}$, we have

$$
h_{x}\left(\left.\Omega_{\widetilde{U}}\right|_{x}\right) \leqslant C \cdot \max _{i} \lambda_{i}^{2}\left(\left.\Omega_{\widetilde{U}}\right|_{x}\right) .
$$

for some constant $C$ depending only on $\operatorname{dim} X$. Therefore, to show that $h\left(\Omega_{\tilde{U}}\right)$ is bounded, it suffices to show that the eigenvalues of $\Omega_{\widetilde{U}}$ are bounded.

Since the manifold $U$ is holomorphic symplectic, its canonicall class is trivial. Denote by $\eta \in \Omega^{\operatorname{dim} X} U$ the trivializing section of the canonical class, obtained as an $\frac{\operatorname{dim} X}{2}$-th power of $\Omega_{X}$. Since the resolution $\pi: \widetilde{X} \longrightarrow X$ is crepant, the section $\eta$ can be extended to a global section $\widetilde{\eta}$ of the canonical class of $\Omega^{\operatorname{dim} X} \tilde{X}$. Therefore, the Hermitian norm $h(\widetilde{\eta})$ is bounded over any compact. On the other hand, for all $x \in \widetilde{U}$, the norm $\left.h(\widetilde{\eta})\right|_{x}$ is equal (up to a canonical constant) to a product of all eigenvalues of $\left.\Omega_{\widetilde{U}}\right|_{x}$. Therefore, $h(\widetilde{\eta})$ is bounded implies $h\left(\Omega_{\tilde{U}}\right)$ is bounded, unless some eigenvalues of $\left.\Omega_{\tilde{U}}\right|_{x}$ tend to zero as $x$ tends to the complement $\widetilde{X} \backslash \tilde{U}$. Therefore, to prove that the eigenvalues of $\Omega_{\widetilde{U}}$ are bounded from above, it suffices to show that these eigenvalues are bounded from below by some positive constant.

Consider the Kähler metric on $U \subset X$ obtained from the flat metric on $V$. The map $\pi: \widetilde{X} \longrightarrow X$ is analytic, and therefore, Lipschitz on compact subsets $K \subset \widetilde{X}$ (the Lipschitz constant being given by the supremum of the absolute value of the derivative). By definition of Lipschitz mappings, for any compact set $K \subset \widetilde{X}$, we may assume that, after rescaling the metric, the map $\left.\pi\right|_{K}$ is decreasing distances.

The form $\Omega_{U}$ is parallel with respect to the natural flat coordinates on $U$. Clearly, the eigenvalues of $\Omega_{U}$ are constant. Since $\pi: \widetilde{U} \longrightarrow U$ is decreasing distances, the eigenvalues of $\Omega_{\widetilde{U}}=\pi^{*} \Omega_{U}$ are bounded from below by a positive constant. As we have shown earlier, this implies that these eigenvalues are bounded from above. 2.4 is proven.

Definition 2.5. Let $\pi: \widetilde{X} \longrightarrow X$ be a resolution of singularities. The map $\pi$ is called semismall if $X$ admits a stratification $\mathfrak{S}$ with open strata $U_{i}$, such that

$$
\forall x \in U_{i} \mid \operatorname{dim} \pi^{-1}(x) \leqslant \frac{1}{2} \operatorname{codim} U_{i}
$$


DEFINITION 2.6. Let $V$ be a symplectic vector space, $G \subset S p(V)$ a finite group, $X=V / G$. For every subgroup $G_{1} \subset G$, consider its fixed point set $V_{G_{1}}$. Let $X_{G_{1}} \subset X$ be the image of $V_{G_{1}}$ under the quotient map $\sigma: V \rightarrow V / G$. Consider a stratification of $X$ with closed strata $X_{G_{1}}$, numbered by the subgroups $G_{1} \subset G$. This stratification is called the $G$-stratification of $X$. There is a similar stratification of $V$, which is also called a $G$-stratification.

The main tool of our arguments is the following theorem.

THEOREM 2.7. Let $\pi: \widetilde{X} \longrightarrow X$ be a symplectic resolution of a quotient singularity $X=V / G, G \in S p(V)$. Then $\pi$ is semismall with respect to the $G$-stratification.

Proof. This statement easily follows from Proposition 4.16 and Proposition 4.5 of [V2] (see also [K], Proposition 4.4).

\section{Symplectic and complex reflections.}

3.1. The statement of the main result. Let $V$ be a symplectic $\mathbb{C}$-vector space.

DEFINITION 3.1. Let $\gamma \in S p(V)$ be an endomorphism of finite order. We say that $\gamma$ is a symplectic reflection if $\operatorname{codim}_{V} V_{\gamma}=2$, where $V_{\gamma}$ is the space of all vectors fixed by $\gamma$.

This is a "symplectic analogue" of the usual notion of a complex reflection. The complex reflection is a linear automorphism of a vector space fixing a subspace of codimension 1. Since the fixed space of a symplectomorphism must be symplectic, the minimal codimension of $V_{\gamma}$ is 2 ; the endomorphism $\gamma$ is a symplectic reflection when this minimum is reached.

We say that a group $G \subset S p(V)$ is generated by symplectic reflection if there exist symplectic reflections $\gamma_{1}, \ldots \gamma_{n} \in G$ which generate $G$.

The main result of this paper is the following

THEOREM 3.2. Let $V$ be a symplectic vector space over $\mathbb{C}$, and $G \subset S p(V)$ a finite group. Assume that the quotient $X=V / G$ admits a symplectic resolution. Then $G$ is generated by symplectic reflections.

The rest of this paper is taken by the proof of 3.2 .

As a first corollary of 3.2 , we obtain a new proof of the following theorem of Kaledin ([K], Theorem 1.7).

THEOREM 3.3. Let $W$ be a complex vector space, $G \subset$ End $W$ a finite group of endomorphisms of $W$, and $V:=W \oplus W^{*}$ be a symplectic space, obtained as a direct sum of $W$ and its dual. Consider the natural embedding $G \hookrightarrow S p(V)$. Assume the $V / G$ has a symplectic resolution. Then the action of $G$ on $W$ is generated by complex reflections.

Proof. By 3.2, the action of $G$ on $V$ is generated by symplectic reflections. Take a symplectic reflection $g \in G$, and let $W_{g}, V_{g}$ be the fixed subspaces of the action of $g$ on $V_{g}, W_{g}$. By definition, $V_{g}=W_{g} \oplus W_{g}^{*}$. Since codim $V_{g}=2$, we have $\operatorname{codim} W_{g}=1$. Therefore, $g$ acts on $W$ as a complex reflection.

Using the arguments of [KV2], Theorem 5.6, one can immediately obtain the following corollary of 3.3, which generalizes [KV2], Theorem 5.6. 
COROLlaRY 3.4. Let $T$ be a 2-dimensional compact complex torus, which is Mumford-Tate generic (see Definition 5.4 of [KV2]). Consider the natural holomorphic symplectic structure on $T$ and its $n$-th power $T^{n}$. Let $G$ be a finite group acting on $T^{n}$ by symplectomorphisms and fixing a point

$$
\hat{x} \in T^{n}, \quad \hat{x}=(\underbrace{x, \ldots, x}_{N \text { times }}), \quad x \in T .
$$

Assume that $T^{n} / G$ admits a symplectic resolution. Then $G$ is a Weyl group associated with some reductive Lie group $\mathfrak{g}$. Moreover, the tangent space $T_{\hat{x}}^{n}$ is identified as a representation of $G$ with $\mathfrak{h} \oplus \mathfrak{h}$, where $\mathfrak{h}$ is the Cartan algebra of $\mathfrak{g}$.

3.2. Groups generated by complex reflections. The proof of 3.2 is based on the same ideas as the proof of the following well-known statement ([Bou], Ch. V, $\S 5$ Theorem 4).

Proposition 3.5. Let $V$ be a complex vector space, and $G \subset G L(V)$ a finite group acting on $V$. Assume that $X:=V / G$ is smooth. Then $G$ is generated by complex reflections.

Proof. Let $G_{0} \subset G$ be a maximal subgroup of $G$ generated by complex reflections. Clearly, $G_{0}$ is a normal subrgroup of $G$. Consider the corresponding quotient $X_{0}:=$ $V / G_{0}$, and let

$$
\tau: X_{0} \stackrel{* /\left(G / G_{0}\right)}{\longrightarrow} X
$$

be the natural quotient map.

Since $G_{0}$ contains all the complex reflections, for all $\eta$ in the complement $G \backslash G_{0}$, the fixed point set $V_{\eta} \subset V$ has codimension $>1$. Let

$$
S:=\bigcup_{\eta \in G \backslash G_{0}} V_{\eta},
$$

and $S_{0}:=S / G_{0} \subset X_{0}$ its image in $X_{0}$. Clearly,

$$
\operatorname{codim}_{X_{0}} S_{0}>1
$$

The following claim is trivial.

Claim 3.1. The group $\Gamma:=G / G_{0}$ acts freely on $X_{0} \backslash S_{0}$

Proof. Let $\gamma \in \Gamma$ be a non-trivial element, and $\widetilde{\gamma}$ an element of $G \backslash G_{0}$ which is mapped to $\eta$ under the natural quotient map. Consider a fixed point $x \in V / G_{0}$ of $\gamma$, and let $v \in V$ be a point mapped to $x$ under the natural quotient map. Then, $\widetilde{\gamma}(v)=g(v)$, where $g \in G_{0}$. We obtain that $v$ is a fixed point of $g^{-1} \widetilde{\gamma}$. Since $g^{-1} \widetilde{\gamma}$ belongs to $G \backslash G_{0}$, we have

$$
v \in V_{g^{-1} \tilde{\gamma}} \subset S=\bigcup_{\eta \in G \backslash G_{0}} V_{\eta} .
$$

Therefore, $\gamma$ has no fixed point outside of $S_{0}=S / G_{0}$.

REMARK 3.2. Notice that in the proof of Claim 3.1 we nowhere used the exact nature of the group $G_{0}$. This means that Claim 3.1 holds for any normal subgroup $G_{0} \subset G$ : the quotient $\Gamma:=G / G_{0}$ acts freely on the set $X_{0} \backslash S_{0}$ defined as above. 
Let $S_{1}:=S / G \subset X$. By Claim 3.1, the natural quotient map

$$
X_{0} \longrightarrow X
$$

is etale over $X \backslash S_{1}$. By (3.1), codim $S_{1}>1$. Since $X$ is smooth and codim $S_{1}>1$, the open embedding $X \backslash S_{1} \hookrightarrow X$ induces an isomorphism on the fundamental group:

$$
\pi_{1}(X)=\pi_{1}\left(X \backslash S_{1}\right)
$$

Consider the scaling map $h_{\lambda}: V \longrightarrow V, \lambda \in \mathbb{C}$ :

$$
h_{\lambda}(v)=\lambda \cdot v \text {. }
$$

Clearly, this map is $G$-equivariant, and hence, can be extended to $X=V / G$. Taking all $\lambda \in \mathbb{R}$, we obtain a map

$$
h: X \times \mathbb{R} \longrightarrow X, \quad h(x, t)=h_{t}(x) .
$$

This map establishes a contraction of $X$ into a point. Therefore, $X$ is contractible, and $\pi_{1}(X)=0$. By $(3.2), \pi_{1}\left(X \backslash S_{1}\right)=0$. This implies that obtained above etale covering

$$
\tau: X_{0} \backslash S_{0} \stackrel{* / \Gamma}{\longrightarrow} X \backslash S_{1}
$$

is trivial. This covering is by construction a Galois covering with the Galois group $\Gamma$; hence, $\Gamma$ is trivial. This proves 3.5.

REMARK 3.3. A converse statement is also true ([Bou], Ch. V, §5 Theorem 4). Namely, let $X$ be a quotient of a vector space by an action of a group generated by complex reflections. Then $X$ is smooth.

4. Symplectic desingularizations and symplectic reflections. In this section, we prove 3.2 .

4.1. Fundamental groups of resolutions of quotient singularities. We use the following theorem, which seems to be well known.

THEOREM 4.1. Let $V$ be a linear space, and $G \subset G L(V)$ a finite group of linear transformations. Consider the quotient $X:=V / G$, and let $\pi: \tilde{X} \longrightarrow X$ be a resolution of singularities. Then the manifold $\tilde{X}$ is simply connected.

Proof. The following proof was suggested by F. Bogomolov.

First of all, we construct a canonical surjection

$$
G \stackrel{p}{\longrightarrow} \pi_{1}(\tilde{X}) \text {. }
$$

This is done as follows. Let $Z \subset V$ be the union of all $G$-strata of codimension $\geqslant 2$ (2.6). For any $x \in V \backslash Z$, the stabilizing subgroup $S t_{x}(G)$ is generated by complex reflections. A quotient of $\mathbb{C}^{n}$ by an action of a group generated by complex reflection is smooth (Remark 3.3). Therefore, the quotient $(V \backslash Z) / G$ is smooth.

Since the map $\pi: \widetilde{X} \longrightarrow X$ is idenity over smooth points of $X=V / G$, we have a canonical open embedding

$$
(V \backslash Z) / G \hookrightarrow \tilde{X}
$$


The following claim is trivial.

Claim 4.1. Let $U \hookrightarrow Y$ be a Zariski open subvariety of a complex variety $Y$. Then we have a natural epimorphism

$$
\pi_{1}(U) \longrightarrow \pi_{1}(Y)
$$

This gives a natural surjective homomorphism

$$
\pi_{1}((V \backslash Z) / G) \rightarrow \pi_{1}(\tilde{X}) .
$$

Since $\operatorname{codim}_{V} Z>1$, we have $\pi_{1}(V \backslash Z)=\pi_{1}(V)$. Therefore, the manifold $V \backslash Z$ is simply connected.

ClaIM 4.2. A quotient of a simply connected manifold $Y$ by an action of a finite group $G$ has a fundamental group which is a quotient of $G$.

Proof. Any covering $\tilde{Y} \stackrel{a}{\longrightarrow} Y / G$ correspond uniquely to a map $Y \stackrel{b}{\longrightarrow} \tilde{Y}$, in such a way that

$$
a \circ b: Y \longrightarrow Y / G
$$

is the quotient map. $\square$

By Claim 4.2, the group $\pi_{1}((V \backslash Z) / G)$ is a quotient group of $G$. Using (4.3), we obtain the surjection (4.1).

For any $g \in G$, denote the corresponding cyclic group by $\langle g\rangle$. Consider a complex line $L_{g} \subset S^{n} V$ in the symmetric power of $V$, such that

(i) $\langle g\rangle$ fixes $L_{g}$, and we have an embedding $\langle g\rangle \hookrightarrow \operatorname{End}\left(L_{g}\right)$

(ii) $\langle g\rangle$ is the group $S t\left(L_{g}\right)$ of all $\gamma \in G$ which map the line $L_{g}$ to itself.

Such a line exists by invariant theory (any representation of $G$ is a component of $S^{n} V$, in particular, an induced representation, corresponding to an embedding $\langle g\rangle \subset G)$.

Let

$$
C_{g}:=L_{g} \backslash\{0\} /\langle g\rangle
$$

be the corresponding subset of $V / G$. Clearly, $C_{g}$ lies in $(V \backslash Z) / G$. As a complex manifold, $C_{g}$ is naturally isomorphic to $\mathbb{C}^{*}$. Denote by $\gamma_{g}$ the generator of $\pi_{1}\left(C_{g}\right) \cong \mathbb{Z}$.

LEMMA 4.2. Consider the natural group homomorphism $\left.\pi_{1}\left(C_{g}\right) \stackrel{\tau}{\longrightarrow} \pi_{1}(V \backslash Z) / G\right)$. Then $\tau$ maps $\gamma_{g}$ to the element $p(g)$ corresponding to $g \in G$ under the epimorphism (4.1).

Proof. Clear from the definition of (4.1).

Return to the proof of 4.1. Let $g \in G$. Consider the corresponding curve $C_{g} \stackrel{i}{\longrightarrow} \tilde{X}$. The map $\tilde{X} \longrightarrow X=V / G$ is proper. Using valuative criterion of properness, we extend the embedding $C_{g} \stackrel{i}{\longrightarrow} \tilde{X}$ to a map $\bar{C}_{g} \stackrel{i}{\longrightarrow} \tilde{X}$, where $\bar{C}_{g} \cong \mathbb{C}$ is the completion of $C_{g}$ in zero. Since $\bar{C}_{g}$ is simply connected, the path $\gamma_{g} \subset C_{g}$ is contractible in $\bar{C}_{g} \subset \widetilde{X}$. This proves 4.1 . 
4.2. A subgroup generated by symplectic reflections. Return to the assumptions of 3.2. Let $G_{0} \subset G$ be a subgroup of $G$ generated by all symplectic reflections in $G \subset \operatorname{End}(V)$. Clearly, $G_{0}$ is a normal subgroup of $G$. We denote by $\widetilde{X}_{0}$ the fibered product

$$
\widetilde{X}_{0}:=\left(V / G_{0}\right) \times_{V / G} \tilde{X}
$$

associated with the Cartesian square

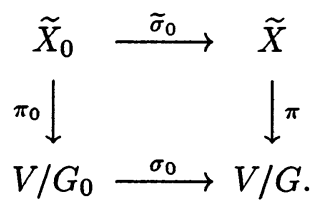

The top horizontal arrow $\widetilde{X}_{0} \stackrel{\widetilde{\sigma}_{0}}{\longrightarrow} \tilde{X}$ is finite, since the bottom horizontal arrow is finite. Denote the ramification variety of the map $\tilde{\sigma}_{0}$ by $R \subset \widetilde{X}$.

LEMMA 4.3. We have $\operatorname{codim}_{\tilde{X}} R \geqslant 2$

Proof. Let $R_{X} \subset X$ be the ramification variety for $\sigma_{0}: \quad V / G_{0} \longrightarrow V / G$. Then $R \subset \pi^{-1}\left(R_{V}\right)$. Clearly, $R_{X}$ is a union of several strata of the $G$-stratification (2.6). By 2.7 , for any stratum $U_{i}$ of a $G$-stratification, we have

$$
\operatorname{codim} \pi^{-1}\left(U_{i}\right) \geqslant \frac{1}{2} \operatorname{codim} U_{1}
$$

Therefore, to prove $\operatorname{codim}_{\tilde{X}} R \geqslant 2$, it suffices to show that

$$
\operatorname{codim}_{X} R_{X} \geqslant 4 \text {. }
$$

Let $Z \subset V$ be the union of fixed sets of all $g \in G \backslash G_{0}$. By Remark 3.2, the group $\left(G / G_{0}\right)$ acts on $(V \backslash Z) / G_{0}$ without fixed points. Therefore, the natural quotient map

$$
(V \backslash Z) / G_{0} \stackrel{\sigma_{0}}{\longrightarrow}(V \backslash Z) / G
$$

is etale. By definition, $R_{X}$ is the ramification variety for the map $\sigma_{0}: V / G_{0} \longrightarrow V / G$. Therefore, $R_{X} \subset \sigma(Z)$, where $\sigma: V \longrightarrow X$ is the quotient map. Since the map $\sigma$ is finite, to prove (4.5) it remains to show that $\operatorname{codim} Z \geqslant 4$. By definition,

$$
Z=\bigcup_{\eta \in G \backslash G_{0}} V_{\eta}
$$

where $V_{\eta}$ denotes a fixed space of $\eta \in G \backslash G_{0}$. To prove $\operatorname{codim} Z \geqslant 4$, we need to show that $\operatorname{codim} V_{\eta} \geqslant 4$. The space $V_{\eta}$ is symplectic, hence its dimension (and codimension) is even. On the other hand, $\operatorname{codim} V_{\eta}>2$, because $\eta$ is not a symplectic reflection. This proves (4.5). We proved 4.3.

Corollary 4.4. The natural embedding $\tilde{X} \backslash R \hookrightarrow \pi_{1}(\tilde{X})$ induces an isomorphism

$$
\pi_{1}(\tilde{X} \backslash R) \cong \pi_{1}(\tilde{X})
$$

Proof. The manifold $\tilde{X}$ is smooth, and $\operatorname{codim}_{\tilde{X}} R>1$ by 4.3 . 
By 4.1 , the manifold $\widetilde{X}$ is simply connected. We obtain that $\widetilde{X} \backslash R$ is also simply connected. On the other hand, the map $\widetilde{\sigma}_{0}$ of (4.4) induces a Galois covering with the Galois group $G / G_{0}$ :

$$
\widetilde{\sigma}_{0}: \widetilde{\sigma}_{0}^{-1}(\tilde{X} \backslash R) \longrightarrow \widetilde{X} \backslash R \text {. }
$$

Since $\tilde{X} \backslash R$ is simply connected, this map is trivial, and its Galois group $G / G_{0}$ is also trivial. This implies that $G$ coincides with its subgroup $G_{0}$ generated by the symplectic reflections. We obtained that $G$ is generated by symplectic reflections. 3.2 is proven.

ACKNOWLEDGEMENTS. This paper appeared in result of fruitful collaborative work with D. Kaledin. F. Bogomolov was very helpful answering my questions; he also suggested the proof of simple-connectedness of $\tilde{X}$ (4.1). I am grateful to V. Batyrev, M. Leenson, A. Kuznetsov, M. Reid and T. Pantev for insightful comments.

\section{REFERENCES}

[BD] Victor V. BATYREV AND Dimitrios I. DAIS, Strong McKay correspondence, Stringtheoretic Hodge Numbers and Mirror Symmetry, alg-geom electronic preprint 9410001, 1994.

[Bea] A. BEAUVIlle, Varietes Kähleriennes dont la première classe de Chern est nulle, J. Diff. Geom., 18 (1983), pp. 755-782.

[Bea2] A. BEAUVILle, Symplectic singularities, alg-geom electronic preprint math.AG/9903079.

[Bou] N. BourbaKI, Groupes et Algèbges de Lie, ch. IV-VI, Hermann, Paris, 1968.

[H] D. HUYBRECHTS, Compact Hyperkaehler Manifolds: Basic Results, alg-geom 9705025.

[IN] Y. ITO AND H. NAKAJIMA, McKay correspondence and Hilbert schemes in dimension three, alg-geom electronic preprint 9803120, 1998.

[IR] Y. ITO AND M. REID, The McKay correspondence for finite subgroups of $S L(3, \mathbb{C})$, alggeom electronic preprint 9411010 (1994), also published in Higher Dimensional Complex Varieties (Trento, June 1994), M. Andreatta, ed., De Gruyter, 1996.

[K] D. KALEDIN, Dynkin diagrams and crepant resolutions of quotient singularities, alg-geom electronic preprint math.AG/9903157.

[KV1] D. KALEDIN AND M. VERBITSKY, Trianalytic subvarieties of generalized Kummer varieties, in Int'l. Math. Res. Not. 9, 1998, pp. 439-461 (also published as alg-geom electronic preprint 9801038).

[KV2] D. KALEDIN AND M. VERBITSKY, Partial resolutions of Hilbert type, Dynkin diagrams and generalized Kummer varieties, alg-geom 9812078.

[N] H. NAKAJIMA, Lectures on Hilbert schemes of points on surfaces, preprint, 1996 (Available at http://www.math.tohoku.ac.jp/tohokumath/nakajima/nakajima.html).

[R] M. REID, McKay correspondence, alg-geom/9702016.

[ST] G. C. ShephaRd AND J. A. TODD, Finite unitary reflection groups, Canad. J. Math., 6 (1954), pp. 274-304.

[V1] M. VERBITSKY, Hyperkähler embeddings and holomorphic holomorphic symplectic structure symplectic geometry II, alg-geom electronic preprint 9403006 (1994) \& GAFA, 5:1 (1995), pp. 92-104.

[V2] M. VERBITKY, Trianalytic subvarieties of the Hilbert scheme of points on a K3 surface, alg-geom/9705004 \& GAFA, 8 (1998), pp. 732-782.

[Y] S. T. YAU, On the Ricci curvature of a compact Kähler manifold and the complex MongeAmpère equation I., Comm. on Pure and Appl. Math., 31 (1978), pp. 339-411. 
